\title{
Study on Application of IGBT-PWM DC Speed Regulating System to Machine Tool Spindle
}

\author{
Chen Guiyin \\ Electric and Electronics School of Wuhan Institute of Shipbuilding Technology \\ Wuhan 430050, Hubei, China \\ Email: 940173762@qq.com
}

\begin{abstract}
In view of rapid development of the high-power DC PWM speed regulating system, this paper adopts the 3G intelligent power module IGBT and INTEL87C196MC CPU of high performance to make technical innovation for the spindle speed regulating system of NC machine tool, and also designs a spindle DC PWM speed regulating system for a large NC machine tool.
\end{abstract}

\section{Keywords-PWM;IPM;IGBT; INTEL87C196MC;CPU}

\section{INTRODUCTION}

Currently, most main motion systems of full-function NC machine tools adopt DC and AC speed regulating spindle motors. The DC motor features good startup and braking performance and applies to smooth speed regulation in the wide range[1][2]. Furthermore, in terms of the closed loop control theory, it is also the foundation of the AC speed regulating system. Usually the traditional DC speed regulating system uses the thyristor - DC motor (V-M) power transmission automatic control system. Along with evolvement of high-power electronic and power devices (e.g., GTR, GTO, P-MOSFET, and IGBT), the high-power DC PWM speed regulating system has developed rapidly. In comparison to the U-M system, the PWM speed regulating system showcases the following strengths[3][4][5]: used;

a) simple main circuit line and less power elements

b) high switching frequency, easy current continuity, less harmonic waves, and low motor loss and heating;

c) good low-speed performance, stable speed, high precision, and wide speed regulating range;

d) wide system frequency band, good dynamic response, and powerful anti-interference capability;

e) main circuit elements operating in the switch state, small conduction loss, and higher device efficiency;

f) higher grid power factor when a three-phase uncontrolled rectifier is used for the DC power supply.

This paper discusses the technical innovation implemented for the spindle speed regulating system of $\mathrm{NC}$ machine tool by using the $3 \mathrm{G}$ intelligent power module IGBT and INTEL87C196MC CPU of high performance.

Figure 1 shows the hardware principle block diagram of the system, which consists of the main circuit and control circuit. The DC power supply is used, and AC can be converted to DC through the common power supply circuit. The control part adopts the 87C196MC SCM. This system is designed for the speed regulating system of a large NC machine tool.

\section{MAIN CIRCUIT PART}

This system is a closed-loop speed regulating system. The current feedback signal is collected using the current Hall sensor, and the rotation speed signal is checked using the tachometer generator. In the actual experiment, greater $\mathrm{AC}$ interference was found in the current feedback signal and voltage feedback signal. The software filtering method, namely, the digital filtering method, was used to reduce the interference of current loop AC component on the basis of not increasing the hardware overhead. The traditional DC servo system implements PWM using two methods: unipolar mode modulation and bipolar mode modulation. The current fluctuation in the bipolar mode is significant, especially in the high power of this system[6][7]. Though the average current is zero in this case, the instantaneous current is not zero. This helps to overcome static friction and improve the system low-speed stability, but the effective value of current is not zero at this moment, which cannot be accepted by the high power motor of this system. Meanwhile, the current fluctuation of PWM in the bipolar mode is doubled in comparison to that in the unipolar mode. In this system, therefore, the output voltage waveform of the adopted PWM in the unipolar mode has only one polarity, which effectively reduces the current fluctuation and also reduces the commutation loss of motor, with the waveform factor close to 1 .

Mitsubishi H-series IGBT intelligent module is designed for the power circuit based on the advanced $3 \mathrm{G}$ IGBT technology and freewheeling diode technology[8]. The policy adopted for the buffer circuit design and thermal design considers the specific applicable motor, and the PM600D - SA060 module can be used. Its rated current value is $600 \mathrm{~A}$, and rated voltage is $600 \mathrm{~V}$. The IPM for the two units, Mitsubishi 3G H series IGBT module with low power consumption, is solid and durable and can be used conveniently. The advanced processing technology ensures low on-state saturation voltage of the $\mathrm{H}$ series IGBT and also keeps the high switching speed required by operation at $20 \mathrm{kHz}$, fully meeting requirements of the spindle system (the selected rated value varies with the power).

Since this system is a high-power speed regulating system operating at the rated voltage, the main circuit current is nearly $30 \mathrm{kw} / 192 \mathrm{~A}(150 \mathrm{~A})$. This system more urgently requires reducing the inductance of power loop because the energy that leads to surge voltage in the power circuit is proportional to $1 / 2 \mathrm{LpI} 2$ (Lp is the parasitic inductance of the bus, and $\mathrm{I}$ is the operating current). For the IGBT module, the external dimension and thermal 
design of the component require a longer power loop connection. If the traditional bus is used to design the circuit, these longer lines will generate more parasitic inductance. This will make the absorbing circuit design difficult. A special bus structure is required to get a kind of low bus inductance circuit suitable for high current operation, namely, the laminated bus structure consisting of the staggered copper plating layer and insulating layer recommended by Mitsubishi is used and can reduce the inductance. The wide plate of laminated bus isolated by the insulating layer is used for bus connection of the positive pole and negative pole. This wide plate blocks the parasitic inductance of power loop. To minimize the bus inductance, the wide and flat positive and negative motherboards connect the IGBT module to the main capacitor bank. Meanwhile, a no-sense capacitor is added to the motherboard to further reduce the parasitic inductance on the line. This speed regulating system implements high-power (30k) DC speed regulation of four quadrants, and the motor is a series-excited DC motor. To meet the forward/reverse rotation requirement of the motor, the armature current direction or the excitation direction must be separately changed because this DC motor is a series-excited motor. If only the voltage direction is changed for the purpose of changing the motor direction, the excitation direction and armature direction change at the same time, but the motor direction is not changed[9]. Therefore, the contactor is used in the main circuit to change the armature current direction and achieve the purpose of direction switching. Since the motor needs to change direction frequently during processing and the contactor is also frequently energized and powered off, the software design ensures that the contactor direction is changed only when the armature current is zero. This aims to protect the contactor and avoid damage to the contactor arising from electric spark when heavy current is switched[10].

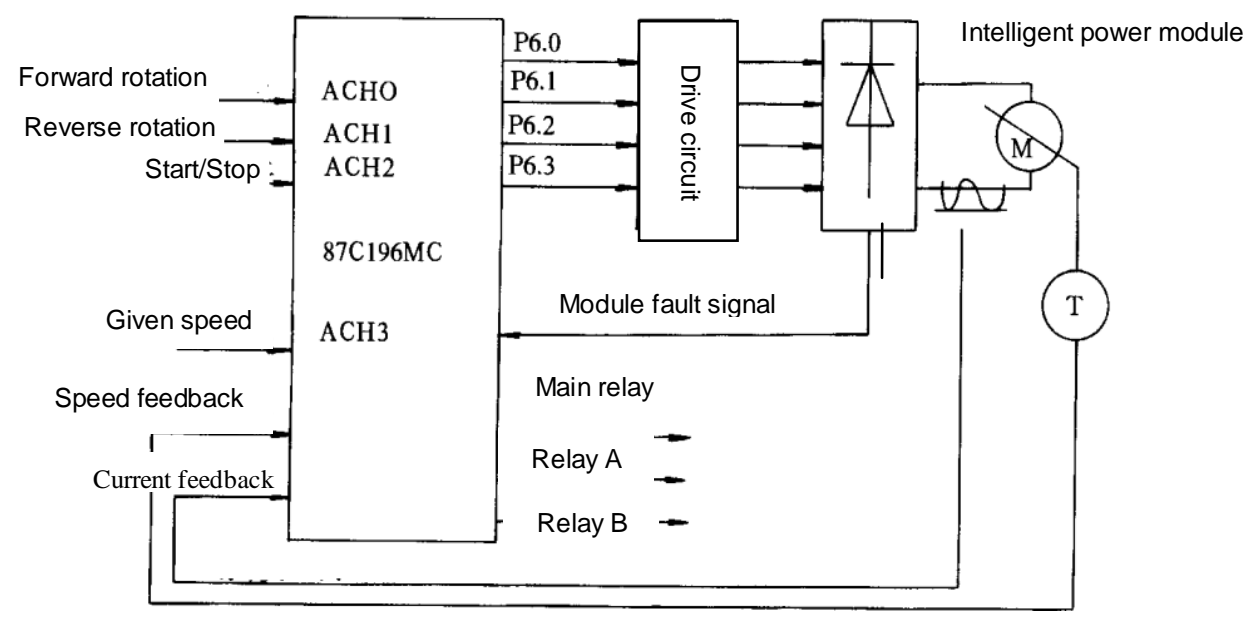

Figure 1. Schematic diagram of the system

\section{CONTROL CIRCUIT PART}

The PWM technology adopts these methods: the CPU, large-scale integrated circuit or the combination of the two. This design selects the CPU method to greatly simplify the control circuit and reduce the components, not only cutting down the cost, but also improving the reliability. The Wave Form Generator (WFG) in the 87C196MC chip is one of its unique characteristics. This peripheral greatly simplifies the software and hardware used to generate synchronous PWM waveform, it especially applies to the PWM of various motors. The WFG has three synchronous PWM modules, each of which contains a phase comparison register, a dead-time generator and a pair of programmable outputs. Once being started, the WFG only requires the CPU to give intervention when the PWM duty ratio is changed. The highly integrated 196 chip is used to reduce complexity of the peripheral interface circuit. Due to the powerful antiinterference capability of the SCM, the WFG can overcome the malicious environment on the field and improve the system performance. Figure 2 shows the block diagram of the main control program.

\section{PROTECTION CIRCUIT}

The protection circuit consists of two main parts: over-current protection of the main circuit; over-current, under-voltage and upper/lower-pipe straight protection. The over-current protection of the main circuit is detected through the current Hall sensor and sent to the SCM. At the same time, the current overload signal is generated through the voltage comparator, and then connected to the external interrupt port. The intelligent module fault signal is generated by the intelligent module. When using the intelligent module fault signal, note that the signal is a non-retention signal. If an upper/lower-pipe fault occurs and the self-protection circuit of the intelligent module takes action, the external input signal will be blocked. Due to the non-retention characteristic, the fault self-protection will be removed after the delay of $\mathrm{T}$, and the external error signal not removed will enter the module again. This repeated fault will damage the module. Therefore, the fault 


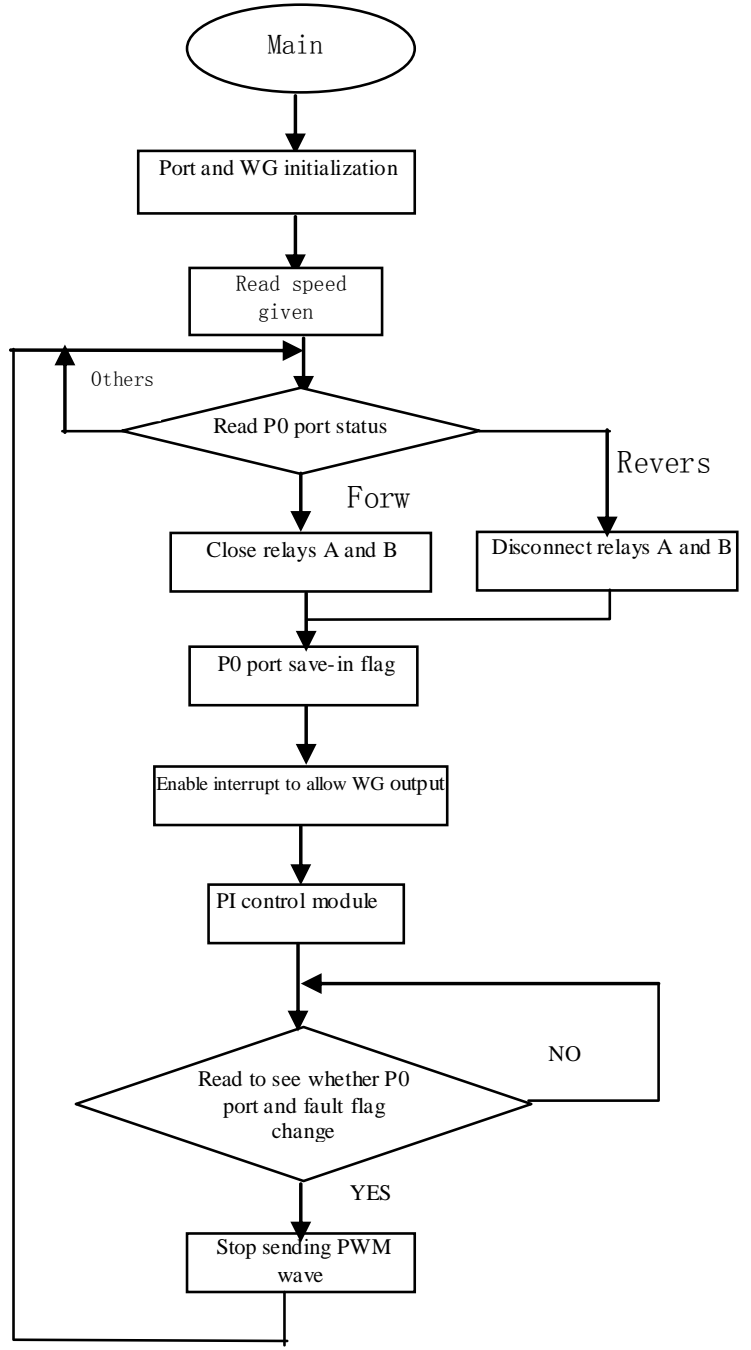

Figure 2. Block diagram of main control program

signal must be handled with caution. In this system, the fault signal is imported to the external interrupt interface of the SCM to perform interrupt handling, and the signal is handled accordingly in the interrupt handler, as shown in Figure 3.

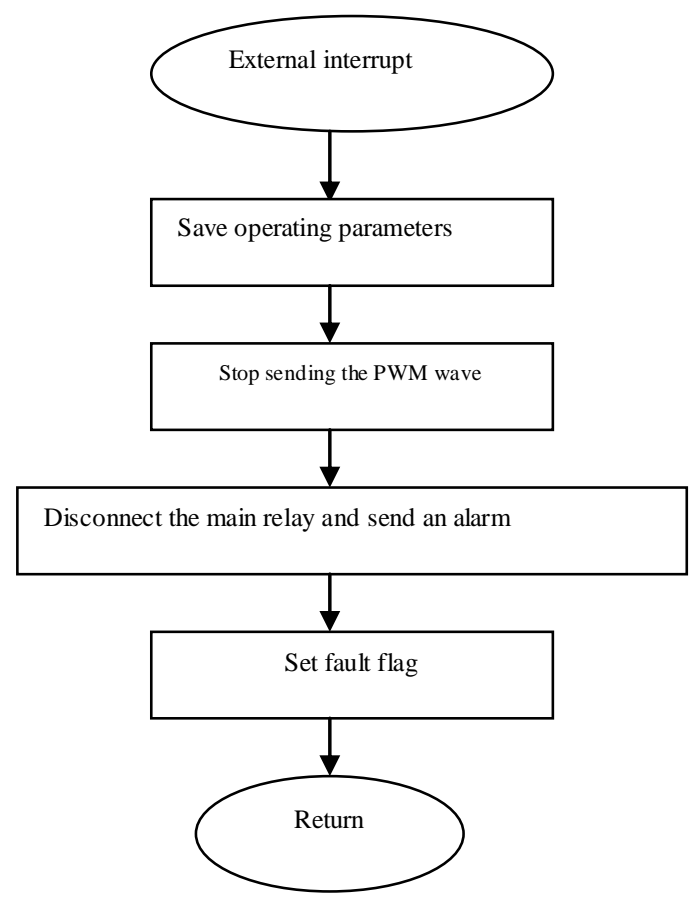

Figure 3. Interrupt handler

\section{CONCLUSION}

This article discusses in detail the application of the PWM DC speed regulating system in the high-power spindle speed regulating system, and studies some special cases to be noted during the design. The design enhances some performances of the original V-M speed regulating system and shows broad application and research prospects in this regard.

\section{ACKNOWLEDGEMENT}

This study is supported by Hubei Provincial Education Science "Twelfth Five Year Plan" 2014 Annual Project (project number: 2014B452); Hubei Province Humanities and Social Sciences project issues (project number: 14Y076).

\section{REFERENCES}

[1] Liu Sheng, Xu Dong-hao, "Intelligent Control for Principal Axis of Variable Vector Propeller of Submersible Vehicle", In Proceeding of 2008 Eighth International Conference on Intelligent Systems Design and Applications, Vol. 2, pp.335 339, 2008.

[2] Li Jun, Kan Shulin, "The Study on Multi-motor Control System Based on Fuzzy PID Control and BP Neural Network", Advances in Information Sciences and Service Sciences, Advanced Institute of Convergence Information Technology, Vol. 4, No. 1, pp. 100 $107,2012$. 
[3] Narbeh Nahapetian, Mohamad Reza Jahed Motlagh, Morteza Analoui, "Adaptive PID Gain Tuning Using Fuzzy Logic and Additional External Performance Index Reference for Controlling Robot Manipulator", In Proceeding of 2009 International Conference on Advanced Computer Control, pp.448 452, 2009.

[4] Qin Yanghao, Wang Donglin. High-power DC PWM Speed Regulating System, Electric Age, 2002, 7: 36 37

[5] Kang Yongzhe, Power Transmission Automatic Control System. Xi'an: Xidian University Press, 1998: 153 163

[6] Li Qingxin, Servo System and Electrical Control of Machine Tool. Beijing: China Machine Press, 1999: 160 165

[7] Feng Duosheng,Application and Maintenance of Frequency Converter. Guangzhou: South China University of Technology Press, 2001: 95 99
[8] Junzhi Yu, Xiaolei Hu, Rui Ding, "Fuzzy Logic PID Based Control Design for Permanent Magnet Synchronous Motor Servo System", In Proceeding of 2009 Second International Conference on Intelligent Computation Technology and Automation, Vol. 2, pp.728 731, 2009.

[9] Leehter Yao, Kian-Leong Lim, "Design of Adaptive Fuzzy PID Controller for Nonlinear System", In Proceeding of 2009 Fourth International Conference on Innovative Computing, Information and Control, pp.565 568, 2009.

[10] Jing Jiang, Shengping Wen, Zhiheng Zhou, Hezhi He, "Fuzzy Barrel Temperature PID Controller Based on Neural Network", In Proceeding of 2008 Congress on Image and Signal Processing, Vol. 1 , pp.90 94, 